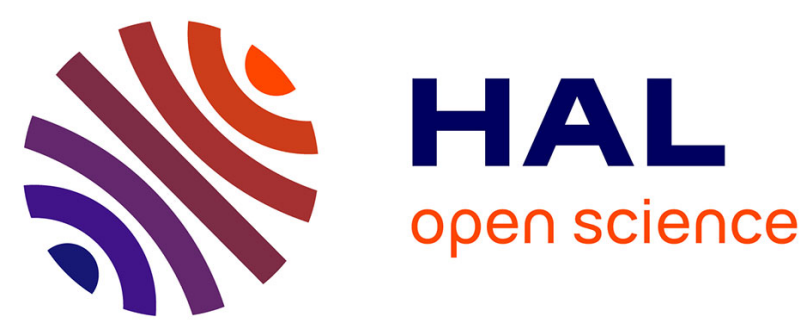

\title{
Cardiac Cine MR Reconstruction From Partial k-Space Using the Notion of Analytic Image.
}

\author{
Josiane Yankam Njiwa, Bassem Hiba, Yue Min Zhu
}

\section{To cite this version:}

Josiane Yankam Njiwa, Bassem Hiba, Yue Min Zhu. Cardiac Cine MR Reconstruction From Partial k-Space Using the Notion of Analytic Image.. Conference proceedings: .. Annual International Conference of the IEEE Engineering in Medicine and Biology Society. IEEE Engineering in Medicine and Biology Society. Annual Conference, 2007, 1, pp.2057-2060. 10.1109/IEMBS.2007.4352725. inserm-00189879

\section{HAL Id: inserm-00189879 https://www.hal.inserm.fr/inserm-00189879}

Submitted on 22 Nov 2007

HAL is a multi-disciplinary open access archive for the deposit and dissemination of scientific research documents, whether they are published or not. The documents may come from teaching and research institutions in France or abroad, or from public or private research centers.
L'archive ouverte pluridisciplinaire HAL, est destinée au dépôt et à la diffusion de documents scientifiques de niveau recherche, publiés ou non, émanant des établissements d'enseignement et de recherche français ou étrangers, des laboratoires publics ou privés. 


\author{
Josiane YANKAM NJIWA, Bassem HIBA and Yuemin ZHU.
}

\begin{abstract}
In the concern of speeding the acquisition time and to increase the spatial resolution of magnetic resonance (MR) images, several methods have already been proposed that acquire partial $k$-space data and use this known information about the imaged object to deal the problem. This work presents a new approach of reconstructing cardiac cine images by using $k$-space redundancies. The proposed method is based on the use of the analytic image concept. It is evaluated by experiments on real human heart images and compared with the Homodyne detection (HM) and the Projection onto convex set (POCS) reconstruction techniques.
\end{abstract}

\section{INTRODUCTION}

$\mathrm{O}$ ne of the main problems in magnetic resonance imaging (MRI) is to shorten the data acquisition time as much as possible while maintaining a good reconstruction quality. In this aim, several methods have been proposed to improve the efficiency of the data acquisition by reducing the amount of acquired data by a given factor referred to as the reduction factor. To reduce the acquisition time in cardiac imaging, fewer methods are available [1]. We are not unaware of the existence of parallel imaging for cardiac cine imaging [2-4] where image reconstruction is made exploiting both temporal correlations and correlations in $k$-space data. Partial $k$-space techniques allow the decrease of the acquisition time and can allow overcoming motion problems in some applications. A fraction of $k$-space is collected only at each time frame for an image sequence and the missing data are then recovered based on the measured $k$-space points from the same time frame. The data recovery technique is based on the principle that each point in $k$-space contains some information about other points. This correlation can be used to recover the missing information. The image at each time frame is reconstructed independently from the images at other time frames. In the present work, a new approach is proposed that exploits redundancies in the $k$-space to perform partial $k$-space reconstruction for cardiac cine imaging. The conventional asymmetric schema of data acquisition is used to exploit the benefits of the analytic

Manuscript received April 2, 2007. Cardiac Cine MR Reconstruction From Partial $k$-Space Using the Notion of Analytic Image.

J. Yankam Njiwa and Y. M. Zhu are CREATIS-LRMN; CNRS, UMR 5220, Villeurbanne, F-69621, France; Inserm, U630, Villeurbanne, F69621, France; INSA-Lyon, Villeurbanne, F-69621, France; Université de Lyon, Lyon, F-69003, France; Université Lyon 1, Villeurbanne, F-69622, France. (e-mail: njiwa@creatis.insa-lyon.fr).

B. Hiba is with ANIMAGE, Rhône-Alpes Genopole, Université Claude Bernard Lyon1, Lyon, France (e-mail: bassem.hiba@cermep.fr). image concept with the knowledge of the high frequencies. This approach provides an increase in imaging speed while being easy to implement and use.

\section{METHOD}

In Homodyne detection (HM) method [5], the $k$-space data is divided in two parts: the low and the high frequencies data. The high known frequencies data are doubled to compensate for the lack of high negatives spatial frequencies data. A ramp function can be used to smooth the transition in the $k$ space. Once the data are smoothed, its inverse Fourier transform is phase-corrected and the desired image is the real part of this phase corrected image.

The projection onto convex sets (POCS) method [6-7] solves the phase compensation problem by iteratively generate the missing data. The phase and the data consistency constraints are considered as two convex sets. One of these sets contains all the images satisfying the predetermined phase constraint, while the other set contains all the images consistent with the measured $k$-space data. The original data are first inverse Fourier transformed to the image domain, where the complex values of the image are projected onto a line that is at an angle equal to the phase estimate. The new image is then Fourier transformed back to the spatial frequency domain where it is used to replace the unknown data. The process is repeated until reaching the last iteration and the desired image is the magnitude of the final obtained spatial image.

\section{Proposed method:}

Although the method presented here uses redundancies in $k$-space to solve the problem of missing data, the main difference between the proposed method and the formers is based on the fact that, the phase estimation step is useless. The use of analytic image concept for static MRI has been presented in our former works [8]. For brevity, we will just introduce some basic equations.

Neglecting noise, a full $k$-space can be expressed as the superposition of its negative and positive spatial frequencies $\operatorname{Sn}(u, v)$ and $S p(u, v)$, respectively in the phaseencoding direction. Let us define the analytic images $z_{p}(x, y)$ and $z_{n}(x, y)$ associated to the positive and the negative half $k$-space, respectively. $z_{p}(x, y)$ has the following expression: 
$z_{p}(x, y)=f_{p}(x, y)+\frac{j}{\pi}$ p.v. $\int_{-\infty}^{+\infty} \frac{f_{p}(\tau, y)}{x-\tau} \mathrm{d} \tau$.

The second analytic image $z_{n}(x, y)$ can be defined in the same way. Finally the analytic image $z(x, y)$ associated to the full $k$-space is given by the expression:

$$
\begin{aligned}
z(x, y) & =f_{p}(x, y)+f_{n}(x, y)+\frac{j}{\pi} \text { p.v. } \int_{-\infty}^{+\infty} \frac{f_{p}(\tau, y)}{x-\tau} \mathrm{d} \tau \\
& +\frac{j}{\pi} \text { p.v. } \int_{-\infty}^{+\infty} \frac{f_{n}(\tau, y)}{x-\tau} \mathrm{d} \tau
\end{aligned}
$$

The asymmetric acquisition schema used for reconstructing images is composed of all the positive $k$-space frequencies together with a few negative frequencies in the phase-encoding direction, as depicted in Fig. 1(a). We split the $k$-space into two parts: the positive and negative $k$-space data, as shown in Figs. 1(b) and 1(c), respectively. The two parts are then considered as the spectral representations of two analytic images.

In this condition, the analytic resultant image given in (2) can be further written as

$$
\begin{aligned}
& z(x, y)=f_{p}(x, y)+f_{n}(x, y)+\frac{j}{\pi} \mathrm{p} \cdot \mathrm{v} \cdot \int_{-\infty}^{+\infty} \frac{f_{p}(\tau, y)}{x-\tau} d \tau+ \\
& \left(\frac{j}{\pi} \mathrm{p} \cdot \mathrm{v} \cdot \int_{-\infty}^{+\infty} \frac{f_{n}(\tau, y)}{x-\tau} d \tau\right) \otimes\left(\frac{1}{2} \delta(x)+\frac{1}{j 2 \pi x}\right)
\end{aligned}
$$

The final reconstructed image, which is the desired image $f$, is obtained by taking the magnitude of $z(x, y)$ in (3).

\section{RESULTS}

The proposed reconstruction method was evaluated on several cardiac cine $k$-space data sets. The $k$-space cine data are raw complex data acquired on a clinical $1.5 \mathrm{~T}$ whole body Siemens Sonata system (Erlangen, Germany). A segmented TRUE FISP sequence was used to acquire ECG-gated cardiac cine images in short and long axe orientations of the heart. For a cardiac cine sequence of $256 \times 176$ pixels, 22 frames per cardiac cycle, and 11 phase encoding lines per segment, 16 cardiac cycles were necessary to achieve a full $k$-space against 10 cardiac cycles for a $5 / 8$ of the $k$-space.

The reconstructed images were compared to the true images reconstructed with the full $k$-space data using both qualitative (difference images) and quantitative (normalized mean square error, and signal to noise ratio criteria) evaluation. The proposed method was also compared with $\mathrm{HM}$ and POCS techniques using the same $k$-space sampling pattern.

The normalized mean square error (NMSE) used for quantitative evaluation is defined by

$$
N M S E=\frac{1}{N M} \sum \sum \frac{\left(I(x, y)-I_{R}(x, y)\right)^{2}}{\bar{I}(x, y) \cdot \bar{I}_{R}(x, y)}
$$

where $I(x, y)$ is the full $k$-space reconstructed image and $I_{R}(x, y)$ the partial $k$-space image reconstructed with the proposed method or HM or still POCS method and $M$ and $N$ are the size of the images.

The blood signal to noise ratio (SNR), denoted as $S N R_{\text {Blood }}$, is calculated as

$S N R_{\text {Blood }}=S I_{\text {Blood }} / S D_{\text {Blood }}$

where $S I_{\text {Blood }}$ and $S D_{\text {Blood }}$ are respectively the mean value and standard deviation of the signal intensity measured in the blood pool.

The myocardium SNR ( $S N R_{\text {Mvocardium }}$ ) is computed as

$S N R$ $=S I_{M}$ $/ S D_{M}$

where $S I_{M}$ and $S D$ are respectively the mean value and standard deviation of the signal intensity measured in the left ventricular myocardium.

Fig. 1 illustrates the results of reconstructing short axis cardiac cine images. Fig.1 (a) to Fig.1 (d) show the frames from the diastolic phase whereas Fig.1 (h) to Fig.1 (k) the frames from the systolic phase. Fig.1 (e) to Fig.1 (g) and Fig.1 (1) to Fig.1 (n) are the corresponding difference images between the full $k$-space reconstructed image and the partial $k$-space reconstructed images. All the time frames are zoomed to the heart. It is seen that the quality of the images reconstructed using the proposed and the POCS methods is closer to that of the reference image. In contrast, with the HM technique, the reconstructed image loses the dynamic range in comparison with that of the reference image, and presents larger errors as shown in TABLE I.

The reconstruction results on long axis cardiac cine images of human heart are shown in Fig. 2, in which Fig. 2 (a) to Fig.2 (d) show the frames from the diastolic phase whereas Fig.2 (h) to Fig.2 (k) the frames from the systolic phase. Fig.2 (e) to Fig.2 (g) and Fig.2 (l) to Fig.2 (n) are the corresponding difference images. The reconstruction error increases with motion during the systolic phase. However, the proposed and the POCS reconstruction methods continue giving similar results with the lowest error values. Again, the HM reconstructed images suffer from loss of dynamic and some edge effects. The HM reconstruction method gives the worst results both qualitatively and quantitatively. The NMSE values are shown in TABLE II.

TABLE III shows mean $\pm S D$ values for $S N R_{\text {Blood }}$, $S N R_{\text {Myocardium }}$. The $t$-tests show that the proposed reconstruction technique resulted in larger $S N R_{\text {Blood }}$ and $S N R_{\text {Myocardium }}$ values than the POCS and the HM techniques. The values obtained by the proposed method are close to those obtained by the full $k$-space reconstruction, followed by the POCS reconstruction. TABLE III also shows that the proposed and POCS methods allow a reconstruction quality which is close to the full $k$-space reconstruction one according to the $P$ 
values whereas the HM method gives results which present significant differences with the full $k$-space results.

\section{CONCLUSION}

The present work proposed a reconstruction method for cardiac cine images using the notion of analytic image. The method presents the particularity of being extremely simple to implement, rather robust and not requiring phase correction. The comparison with the HM and the POCS methods shows that the proposed method yields better reconstruction results than the HM technique both qualitatively (visually) and quantitatively (NMSE, SNR criteria). The results obtained with the POCS method are close to those obtain with the proposed method. The proposed method allows achieving a factor of acquisition time reduction of about 2 .

\section{REFERENCES}

[1] Suga M.; Matsuda T.; Komori M. "Keyhole Method for High-Speed Human Cardiac Cine MR Imaging”. J Magn. Reson. Imag, Vol. 10, pp.778-783, 1999.

[2] Madore B.; Glover G.H.; Pelc N.J. "Unaliasing by Fourier-encoding the overlaps using the temporal dimension (UNFOLD), applied to cardiac imaging and fMRI". Magn. Reson. Med, Vol. 42, pp. 813828, 1999.

[3] Epstein F.H.; Kellman P.; McVeigh E.R. (2000) "First-pass cardiac MRI using UNFOLD”. Radiol. Soc. North. Am, C14:379.

[4] Tsao J.; Boesinger P.; Pruessmann P. " $k-t$ BLAST and $k-t$ SENSE: Dynamic MRI with High Frame Rate Exploiting Spatiotemporal Correlations". Magn. Reson. Med, Vol. 50, pp. 1031-1042, 2003.

[5] Noll D.C.; Nishimura D.G.; Macovski A. "Homodyne detection in magnetic resonance imaging.;" IEEE Trans. Med. Imag, Vol. 10, pp.154-163, 1991.

[6] McGibney G.; Smith M.R Nicholas S.T and Crawley A. "Quantitative evaluation of several partial-Fourier reconstruction algorithms used in MRI”. Magn. Reson. Med, Vol.30, pp. 51-59, 1993.C. J. Kaufman, Rocky Mountain Research Lab., Boulder, CO, private communication, May 1995.

[7] Liang Z.P and Lauterbur P.C. "Principles of Magnetic Resonance Imaging A Signal Processing Perspective", IEEE Press, 2000.

[8] Yankam Njiwa J.; Zhu Y.M.; Robini M. and Magnin I. "Magnetic resonance image reconstruction using the notion of analytic image". Nucl. Inst. Meth. Phys A, Vol. 571, pp. 73-76, 2007.

[9] Hiba B.; Richard N.; Janier M., Croisille P. "Cardiac and Respiratory Double Self- Gated Cine MRI in the Mouse at 7 T". Magn. Reson. Med, Vol. 55, pp. 506-51, 2006.
TABLE I

NMSE ERROR VALUES FOR THE RESULTS IN FIG.1

\begin{tabular}{lcc}
\hline \hline & Diastole & Systole \\
\hline HM & 0.6255 & 0.8932 \\
POCS & 0.0321 & 0.0412 \\
$\begin{array}{l}\text { Our } \\
\text { method }\end{array}$ & 0.0317 & 0.0394
\end{tabular}

TABLE II

NMSE ERROR VALUES FOR THE RESULTS IN FIG. 2

\begin{tabular}{lll}
\hline \hline & Diastole & Systole \\
\hline HM & 0.6631 & 0.8409 \\
POCS & 0.0182 & 0.0509 \\
$\begin{array}{l}\text { Our } \\
\text { method }\end{array}$ & 0.0184 & 0.0488
\end{tabular}

TABLE III

MEAN \pm STANDARD DEVIATION VALUES OF SNR MEASURED IN THE FULL $K$-SPACE, HM, POCS AND THE PROPOSED RECONSTRUCTED IMAGES, RESPECTIVELY. IN THE BRACKETS ARE INSCRIBED THE $P$ VALUES WHICH REPRESENT THE VALUES OF THE $T$-TEST BETWEEN THE FULL $K$-SPACE AND THE PARTIAL $K$-SPACE RECONSTRUCTED IMAGES.

\begin{tabular}{|c|c|c|}
\hline & $S N R_{\text {Blood }}$ & $S N R_{\text {Myocardium }}$ \\
\hline $\begin{array}{l}\text { Full } \\
\text { kspace }\end{array}$ & $11.19 \pm 5$ & $2.77 \pm 0.8$ \\
\hline HM & $\begin{array}{r}9.9 \pm 1.9 \\
(0.0275)\end{array}$ & $\begin{array}{c}2.11 \pm 0.5 \\
(0.0236)\end{array}$ \\
\hline POCS & $\begin{array}{c}12.9 \pm 3.6 \\
(0.0826)\end{array}$ & $\begin{array}{c}2.62 \pm 0.7 \\
(0.1378)\end{array}$ \\
\hline $\begin{array}{l}\text { Our } \\
\text { method }\end{array}$ & $\begin{array}{r}13 \pm 4.12 \\
(0.0731)\end{array}$ & $\begin{array}{c}2.65 \pm 0.67 \\
(0.1786)\end{array}$ \\
\hline
\end{tabular}




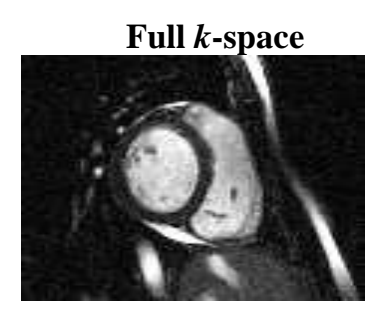

(a)

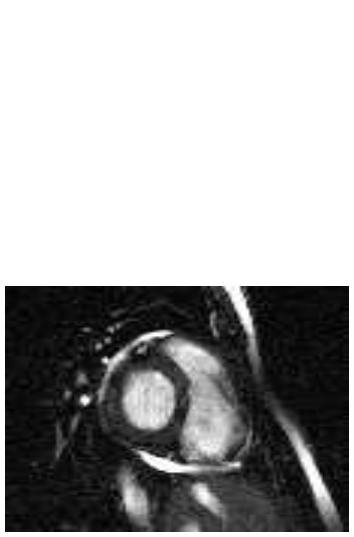

(h)

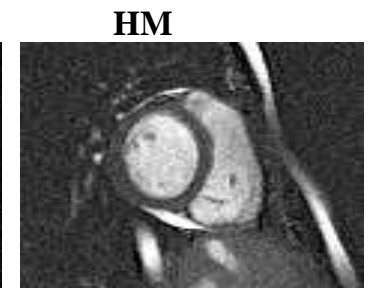

(b)

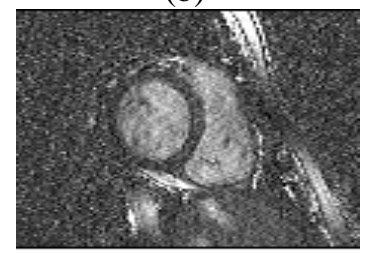

(e)

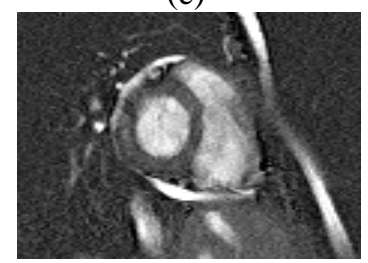

(i)

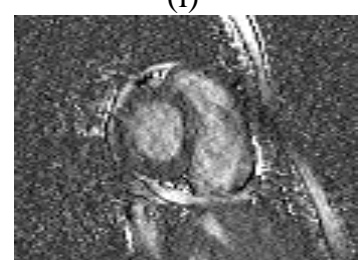

(1)

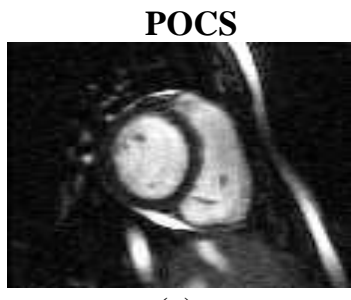

(c)

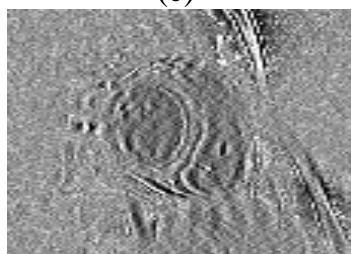

(f)

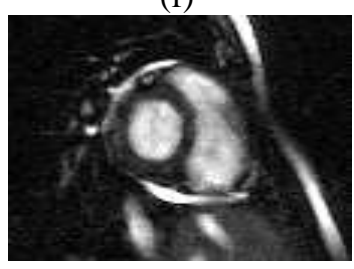

(j)

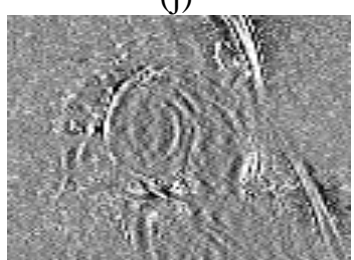

(m)

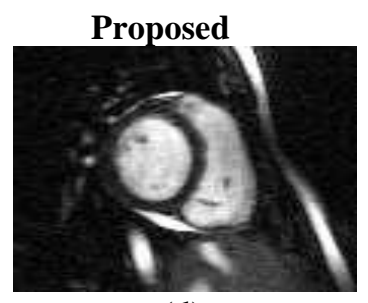

(d)

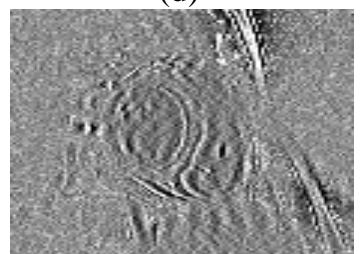

(g)

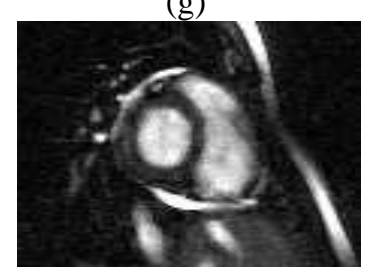

(k)

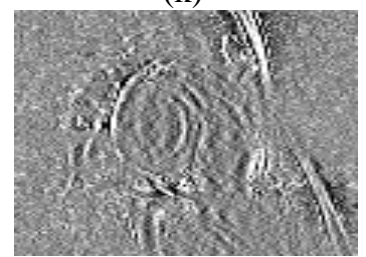

(n)

Fig.1. Reconstruction results on human heart. End diastole (a) Reference image reconstructed from the full $k$-space. (b)-(d) Images reconstructed from partial $k$-space using respectively HM, POCS and the proposed methods. (e)-(g) Difference images between the reference image and images reconstructed using HM, POCS and the proposed method, respectively. End systole (h) Reference image reconstructed from the full $k$-space. (i)-(k) Images reconstructed from partial $k$-space using respectively HM, POCS and the proposed methods. (1)-(n) Difference images between the reference image and images reconstructed using HM, POCS and the proposed method, respectively.

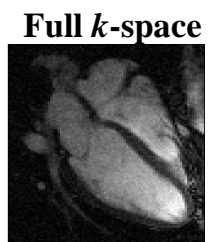

(a)
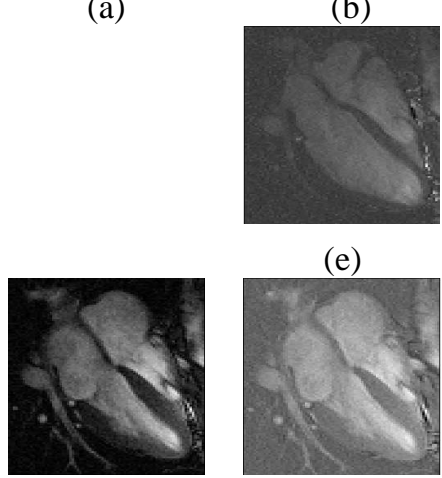

(h)

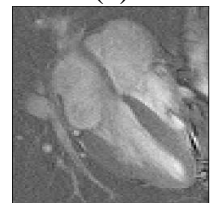

(e)

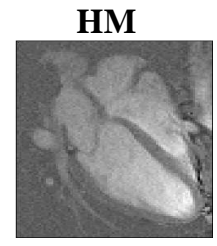

(b)

(i)

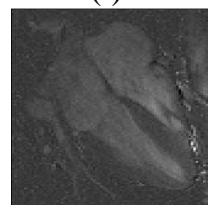

(1)

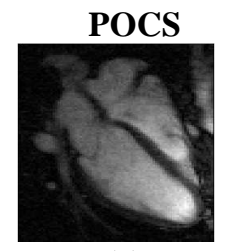

(c)

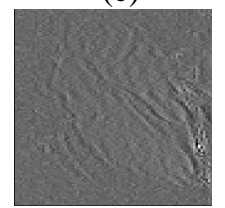

(f)

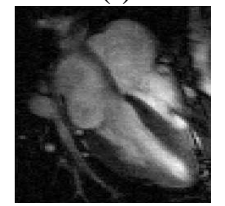

(j)

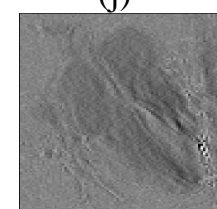

(m)

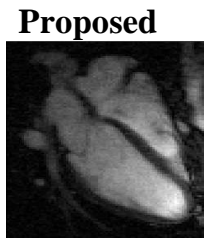

(d)

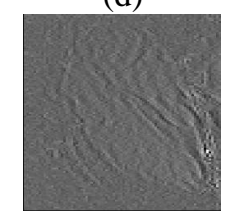

(g)

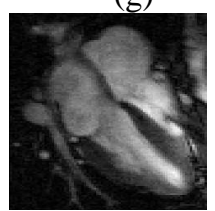

(k)

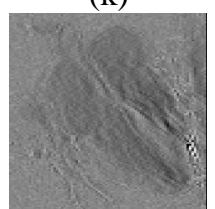

(n)

Fig.2. .Long axis cardiac cine Reconstruction results on human heart. End diastole (a) Reference image reconstructed from the full $k$-space. (b) - (d) Images reconstructed from partial $k$-space using respectively HM, POCS and the proposed methods. (e)(g) Difference images between the reference image and images reconstructed using HM, POCS and the proposed method, respectively. End systole (h) Reference image reconstructed from the full $k$-space. (i)-(k) Images reconstructed from partial $k$ space using respectively HM, POCS and the proposed methods. (1)-(n) Difference images between the reference image and imaoes reconstructed usino HM POCS and the nronosed method resnestivelv 